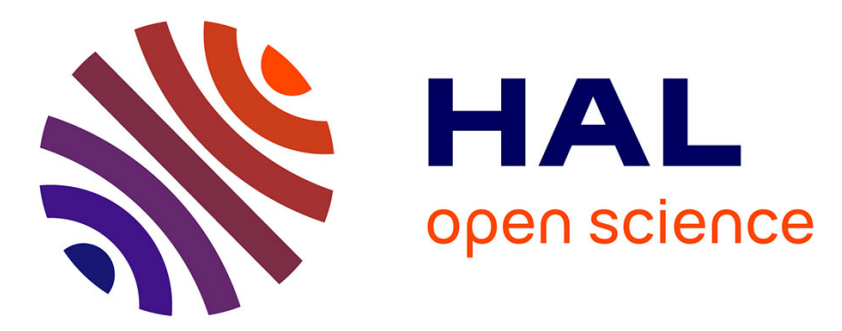

\title{
Critical Properties of a Random-Anisotropy System a - DyNi: A New Universality Class in Disordered Systems?
}

B. Dieny, B. Barbara

\section{To cite this version:}

B. Dieny, B. Barbara. Critical Properties of a Random-Anisotropy System a - DyNi: A New Universality Class in Disordered Systems?. Physical Review Letters, 1986, 57 (9), pp.1169 - 1172. 10.1103/PhysRevLett.57.1169 . hal-01660042

\section{HAL Id: hal-01660042 https://hal.science/hal-01660042}

Submitted on 9 Dec 2017

HAL is a multi-disciplinary open access archive for the deposit and dissemination of scientific research documents, whether they are published or not. The documents may come from teaching and research institutions in France or abroad, or from public or private research centers.
L'archive ouverte pluridisciplinaire HAL, est destinée au dépôt et à la diffusion de documents scientifiques de niveau recherche, publiés ou non, émanant des établissements d'enseignement et de recherche français ou étrangers, des laboratoires publics ou privés. 


\title{
Critical Properties of a Random-Anisotropy System $a$-DyNi: A New Universality Class in Disordered Systems?
}

\author{
B. Dieny and B. Barbara \\ Laboratorie Louis Néel, Centre National de la Recherche Scientifique, Université Scientifique, \\ Technologique et Médicale de Grenoble, 38042 Grenoble Cedex, France \\ (Received 7 August 1985)
}

\begin{abstract}
The scaling properties of a random-anisotropy system (amorphous DyNi) are given for the first time. The phase transition is shown to be of the spin-glass type, with a divergence of the nonlinear magnetization. However, critical exponents are different from those of canonical Heisenberg spinglasses (e.g., $C u \mathrm{Mn}$ or $a$-GdAl). This result strongly suggests that random-anisotropy systems belong to a different universality class. This allows us to understand the scatter observed in the exponents of different Heisenberg spin-glasses as caused by a hyperscaling crossover near the freezing temperature.
\end{abstract}

PACS numbers: $75.40 . \mathrm{Fa}$

In this paper, we present the first measurements and analysis of the nonlinear magnetization of a randomanisotropy system. The singular behavior of the nonlinear magnetization is clearly shown; a scaling analysis yields a coherent set of critical exponents. A comparison of these exponents with those of canonical Heisenberg spin-glasses strongly suggests that random-anisotropy systems belong to a different universality class; as a consequence we attribute the scatter observed in critical exponents of usual spin-glasses (Mn- or Gd-based systems) to a crossover from random exchange to random anisotropy, real spin-glasses always containing superimposed weak randomanisotropy fields arising from higher-order terms in crystal field, electron scattering, etc.

The present study is performed on the amorphous system $\mathrm{DyNi}_{1.33}$. This alloy can be considered as a good realization of the random-anisotropy model, as

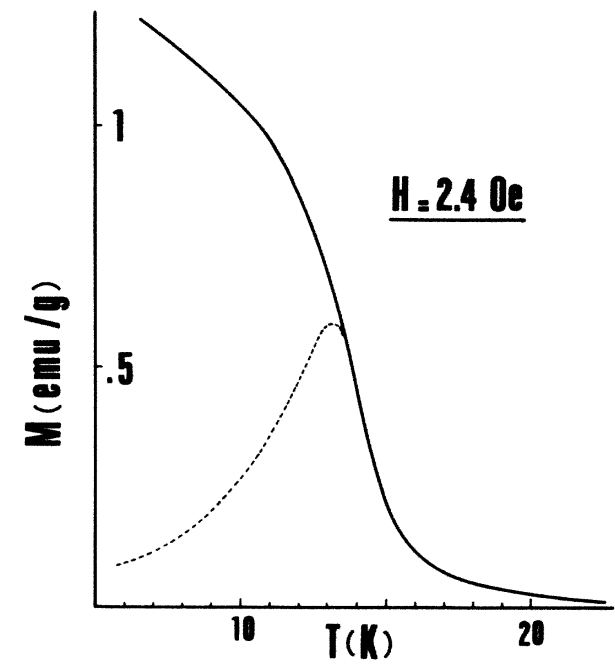

FIG. 1. Zero-field-cooled (dashed line) and field-cooled (solid line) magnetization of $a-\mathrm{DyNi}$. shown elsewhere. ${ }^{1}$ Zero-field and field-cooled magnetization measurements have been performed for a wide range of temperatures $(4.2$ to $30 \mathrm{~K})$ and fields ( 2.4 to $150 \mathrm{Oe}$ ) with a dc SQUID magnetometer where the temperature is determined to $\pm 5 \times 10^{-3} \mathrm{~K}$ and the field to $\pm 1 \%$ in the range of a few oersteds and $\pm 0.5 \%$ in the range of $10^{2}$ Oe. A typical curve is given Fig. 1. Notice that, in contrast to Heisenberg spin-glasses, the field-cooled curve has no maximum.

The initial susceptibility has been obtained from zero-field extrapolation of the isothermal lines $m / h \mathrm{vs}$ $h$ obtained for different temperatures (Fig. 2) where $m$ is the measured magnetization and $h$ the magnetic field. At first sight $\chi_{0}(T)$ looks like the susceptibility

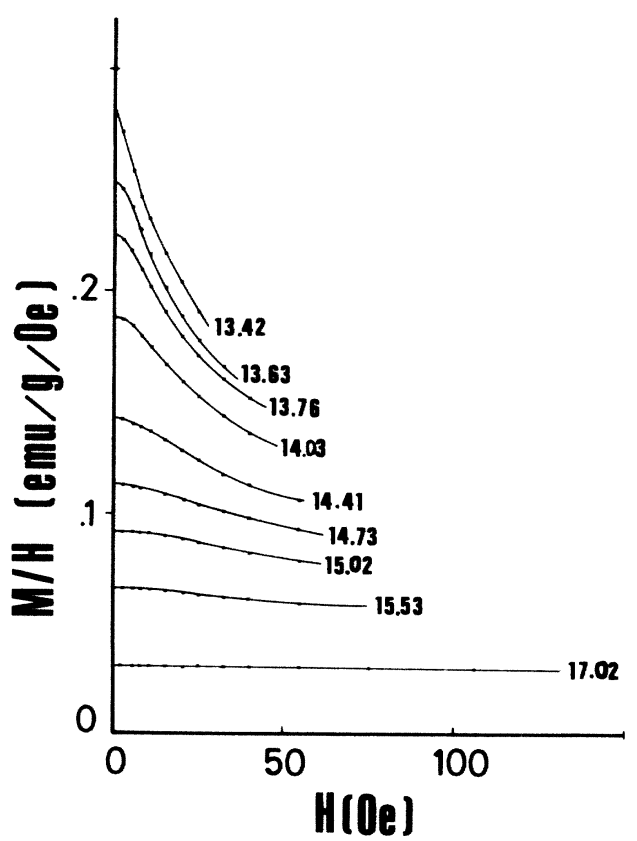

FIG. 2. Plot of $m / h$ vs $h$ in $a$-DyNi for different temperatures between 13.42 and $17.02 \mathrm{~K}$. 
of a ferromagnet with a somewhat broadened transition (Fig. 3).

Starting from Suzuki's expression of the free energy ${ }^{2}$

$$
F(m, q)=\frac{1}{2} a(1+q) m^{2}+c q^{2} G\left(q t^{-\beta}\right)-m h,
$$

the initial susceptibility can be expressed as

$$
\chi_{0}=1 / a(T)(1+q),
$$

where $q$ is the Edwards-Anderson parameter and $a(T)$ describes the ferromagnetic correlations. Two cases must be considered, above and below $T_{f}$. (i) Above the transition temperature $T_{f}, q=0$ and $\chi_{0}=\chi_{0}^{0}$ $=a^{-1}(T)$. The fit to our data shows a crossover on $a(T)$ for decreasing temperatures from a regime in $t^{-\gamma_{0}}\left[t=\left(T-T_{f}\right) / T_{f}\right.$ with $T_{f}=13.5 \mathrm{~K}$ and $\gamma_{0}=1.3$ \pm 0.2 , as in a ferromagnet without disorder] to a regular regime closer to the transition $(13.5<T<16 \mathrm{~K}$, which corresponds to a freezing of ferromagnetic correlations). In this second regime, $\chi_{0}=\chi_{0}^{0}=(T$ $-10.5)^{-2.7}$. (ii) Below $T_{f}, \chi_{0}=\chi_{0}^{0}\left(1-t^{\beta}\right)^{3}$ Extrapolating the form $\chi_{0}^{0}=(T-10.5)^{-2.7}$, some degrees below $T_{f}$, we find $\beta=1.2 \pm 0.2$ (Fig. 3 ). It is important to note that, within experimental errors, the transition temperature obtained from Fischer's relation corresponds with the temperature for which a hysteresis appears in zero-field-field-cooled experiments (Fig. 1).

Now let us consider the nonlinear susceptibility $\chi_{S}=\chi_{0}-m / h$. From (1), it can be written

$$
\frac{x_{s}}{x_{0}}=\left(\frac{m^{2}}{x_{0}}\right)^{1 / \delta} f\left(\frac{m^{2}}{x_{0}} t^{-\phi}\right) \text {. }
$$

Here, the approximation generally used in spin-glasses according to which $m=\chi_{0} h=h / T_{f}$ in the critical re-

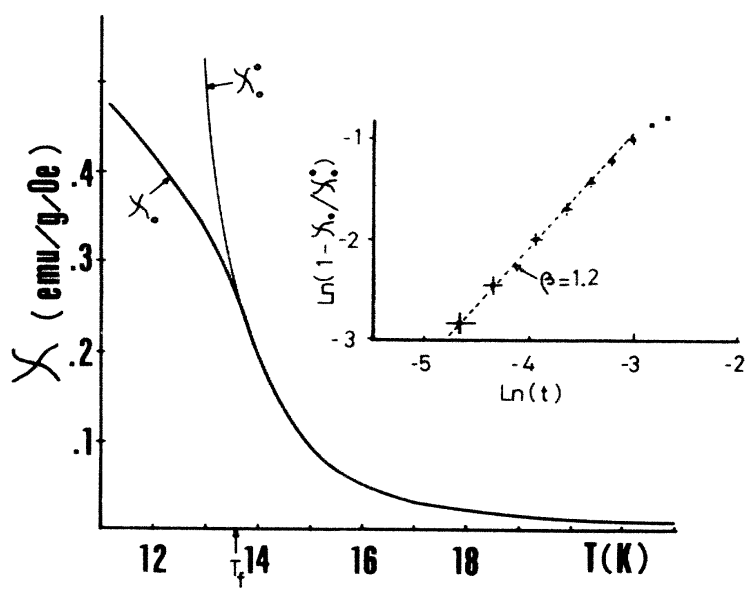

FIG. 3. Thermal variations of the measured susceptibility $\chi_{0}$ of $a-\mathrm{DyNi}$ and of the calculated $\chi_{8}$ curve (see text). Inset: Log-log plot of the nonregular zero-field susceptibility $\left(\chi_{8}-\chi_{0}\right) / \chi_{8}^{8}$ vs the reduced temperature $t$ in $a-\mathrm{DyNi}$. gime cannot be used as a result of the drastic increase of the regular magnetization near $T_{f}$ (see Fig. 3). Therefore, we have plotted our nonlinear susceptibility $\chi_{s}$ according to expression (3), where $\chi_{0} h$ is the measured linear magnetization and $t=(T-13.5) / 13.5$ the reduced temperature, for different values of $\delta$ and $\phi$. The best fit has been obtained for $\delta=2.7 \pm 0.1$ and $\phi=3.2 \pm 0.1$ in field and reduced temperature ranges $2.4<h<100$ Oe and $0.005<t<0.25$ (Fig. 4). Incidentally, the exponent $\delta$ given here is not simply related to the one calculated by Aharony and Pytte 4 in their earlier theory. The other exponents can be derived from usual scaling relations; $\beta=\phi / \delta$ gives $\beta=1.2 \pm 0.1$, in agreement with the value $1.2 \pm 0.1$ determined independently from the Fischer relation, below $T_{f}$. On the other hand, if one believes the conjecture according to which the irreversibility line is related to the crossover exponents $\phi$, through the relation $h^{2} \propto t^{\phi},{ }^{5-7}$ we would have a second check for the value obtained for $\phi$ : In this analysis $\phi=3.2 \pm 0.1$ whereas from the crossover line $\phi=3.1 \pm 0.1{ }^{1}$ The exponent $\gamma$ of the first nonlinear susceptibility term can be evaluated from the equality $\gamma=\phi(\delta-1) / \delta$; we find $\gamma=2 \pm 0.1$.

As shown Table I, critical exponents of the random-anisotropy system $a$-DyNi are different from those of canonical Heisenberg spin-glasses such as $a$ $\mathrm{GdAl}$ or $\mathrm{CuMn}$. Nevertheless, the differences are not so marked with other systems such as $A g \mathrm{Mn}$ where $\gamma=2 .{ }^{10}$ In fact, since the experimental demonstration of the critical behavior of the nonlinear susceptibility of spin-glasses, ${ }^{8}$ critical exponents of many different systems have been published and now it appears that they are not all the same, in spite of error bars. ${ }^{11}$ However, such a scatter can be considerably reduced if one assumes the coexistence of two main types of spin-glasses: (i) the $a$-GdAl type where typically $\gamma=3 \pm 0.1$ and $\delta \simeq 5.5 \pm 0.5$, and (ii) the $A g \mathrm{Mn}$ type where $\gamma=2 \pm 0.1$ and $\delta \simeq 2.5 \pm 0.5$. Interestingly, the

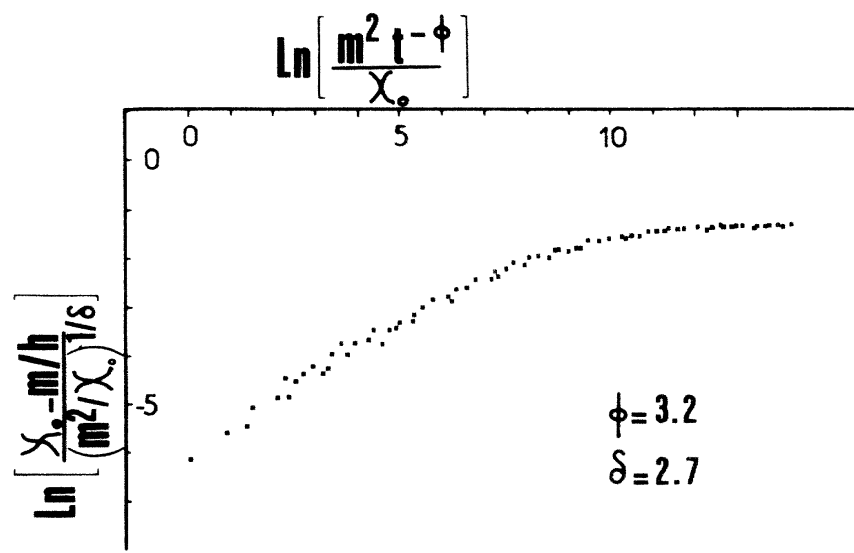

FIG. 4. Scaling plot of the nonlinear susceptibility of $a$ DyNi. 
TABLE I. Comparison of critical exponents measured from dc experiments. The infinite-cluster dimensionality $D_{f}$ is calculated from the hyperscaling expression $D_{f}=d \phi /(\phi+\beta)$ for $d=3$. The exponent $\eta$ characterizing deviations from the Ornstein-Zernike form of correlations is obtained from $2-\eta=\gamma d /(\phi+\beta)$. A dimensionality crossover appears between systems classified number 1 and number $2\left(D_{f}=2.5\right.$ to $\left.D_{f}=2.2\right)$. Heisenberg spin-glasses behave almost like randomanisotropy systems when $t_{\max } \leqslant 0.1$ (class $2 / 1$ ).

\begin{tabular}{|c|c|c|c|c|c|c|c|c|c|}
\hline & $\phi$ & $\delta$ & $\gamma$ & $\beta$ & $\nu$ & $\eta$ & $t_{\max }$ & $D_{f}$ & Class \\
\hline $\operatorname{CuMn}(4.6 \%)^{\mathrm{a}}$ & $5 \pm 1$ & $4.2 \pm 0.1$ & $3.4 \pm 0.4$ & $1.1 \pm 0.2$ & $2 \pm 0.4$ & 0.30 & 2 & 2.4 & 1 \\
\hline $\operatorname{CuMn}(1 \%)^{\mathrm{b}}$ & $4 \pm 1$ & $5.7 \pm 0.5$ & $3.3 \pm 0.5$ & 0.7 & $1.6 \pm 0.4$ & -0.06 & 1 & 2.5 & 1 \\
\hline $\operatorname{CuMn}(0.25 \%)^{\mathrm{c}}$ & $4.6 \pm 1$ & $4.6 \pm 0.3$ & $3.6 \pm 0.3$ & 1 & $1.9 \pm 0.4$ & 0.10 & 0.7 & 2.5 & 1 \\
\hline$a-\mathrm{GdAl}^{\mathrm{d}}$ & $3.3 \pm 0.4$ & $5.7 \pm 0.2$ & $2.7 \pm 0.1$ & 0.7 & $1.3 \pm 0.2$ & 0.08 & 0.15 & 2.5 & 1 \\
\hline $\mathrm{CsNiFeF}_{6}{ }^{\mathrm{e}}$ & $4.2 \pm 0.5$ & $3.5 \pm 0.5$ & $3.0 \pm 0.5$ & $1.2 \pm 0.1$ & $1.8 \pm 0.2$ & 0.33 & 0.1 & 2.3 & $2 / 1$ \\
\hline $\operatorname{AgMn}(0.4-20 \%)^{f}$ & $3.3 \pm 0.2$ & $3.1 \pm 0.2$ & $2.2 \pm 0.2$ & $1 \pm 0.1$ & $1.3 \pm 0.1$ & 0.46 & 0.1 & 2.3 & $2 / 1$ \\
\hline$a-\mathrm{DyNi}$ & $3.2 \pm 0.1$ & $2.7 \pm 0.1$ & $2.0 \pm 0.1$ & $1.2 \pm 0.1$ & $1.5 \pm 0.1$ & 0.63 & 0.25 & 2.2 & 2 \\
\hline
\end{tabular}

aReference 8 .

bR. Omari, J. J. Préjean, and J. Souletie, J. Phys. (Paris) 44, 1069 (1983).

cA. Berton, J. Chaussy, J. Odin, R. Rammal, and R. Tournier, J. Phys. (Paris), Lett. 43, L153 (1982).

${ }^{\mathrm{d}}$ References 8 and 9.

eP. Beauvillain, C. Chappert, and J. P. Renard, J. Phys. (Paris), Lett. 45, L665 (1984); P. Beauvillain, C. Dupas, J. P. Renard, and P. Veillet, Phys. Rev. B 29, 4086 (1984).

fReference 10.

gThis work.

exponents found for the random-anisotropy system $a$ DyNi, $\gamma=2 \pm 0.1$ and $\delta=2.7 \pm 0.1$, are very close to those of the AgMn type. All these observations become coherent within the following scenario:

We admit, first, that Heisenberg spin-glasses ( $a$ $\mathrm{GdAl}$ type) and random-anisotropy systems ( $a$ - $\mathrm{DyNi})$ do not belong to the same universality class (which is not surprising since their random character issues from different random fields), and second that usual spinglasses must contain, to some extent, weak randomanisotropy fields of diverse origin (crystal-field effects on $5 d$ electrons for Gd-based systems, or other highorder types of anisotropies in Mn-based systems, dipolar effects, etc). Therefore usual spin-glasses should present, when the temperature approaches $T_{f}$, a crossover from Heisenberg spin-glass to random-anisotropy exponents, similar to the Heisenberg-to-Ising crossover observed in ferromagnets where a weak uniform anisotropy is always present (even in $S$ type of ions). In fact a similar crossover has been predicted in spinglasses by Kotliar and Sompolinsky ${ }^{12}$ in a mean-field model. Recently, Campbell, de Courtenay, and Fert ${ }^{13}$ have interpreted irreversibility lines of CuMn alloys in terms of such a crossover. Furthermore, in two very recent studies, Yeshurun and Sompolinsky ${ }^{14}$ and de Courtenay et al. ${ }^{15}$ have shown that the spin-glass critical exponents of $A g \mathrm{Mn}$ correspond to anisotropydominated conditions, as is predicted in the present scenario.

Finally, let us give a scaling approach for the spinglass to random-anisotropy crossover. Generalizing expression (1) of Aharony and Pytte, ${ }^{4}$ we express the magnetization as a function of a random field $\Delta$ :

$$
m=|t|^{\beta_{0}} f\left(h|t|^{-\phi_{0}}, \Delta|t|^{-\phi_{\Delta}}\right),
$$

where $t=\left[T-T_{f}(\Delta)\right] / T$.

In the particular case of a random-anisotropy system $\Delta=(D / J)^{2}$ is such that $T_{f}(0)=T_{c}$ and therefore $\beta_{0}$ and $\phi_{0}$ are the critical exponents of a ferromagnet. For a spin-glass $\Delta=\left(J / J_{0}\right)^{2}$ is the variance of the distribution of exchange interactions.

If $\Delta \neq 0$, expression (4) may be written, for $\Delta<<|t|^{\phi_{\Delta}}$,

$$
m=\frac{h|t|^{\beta_{\Delta}}}{\Delta} g_{1}\left(h^{2}|t|^{-2 \phi_{0}}\right)
$$

with

$$
\beta_{\Delta}=\phi_{\Delta}-\gamma_{0}
$$

for $\Delta>\mid t^{\mid \phi} \Delta$,

$$
m=\Delta^{\beta_{0} / \phi_{\Delta}} g_{2}\left(h \Delta^{-\phi_{0} / \phi_{\Delta}}\right) \text {. }
$$

For a given type of random field $\Delta$, the exponents $\phi_{0}$ and $\phi_{\Delta}$ characterize different types of crossover: first, the crossover when $h^{2}=t^{2 \phi_{0}}$, between field- or temperature-dominated regions, which has been already observed in spin-glasses ${ }^{9,16}$, second, the crossover when $\Delta=t^{\phi_{\Delta}}$ between thermal critical fluctuations and static fluctuations of the random field. These last fluctuations can be dominated by the applied field giving rise to a third crossover when $H=\Delta^{\phi_{0} / \phi_{\Delta}}$.

In a real Heisenberg spin-glass, random-anisotropy fields should be relevant near $T_{f}$ if

$$
(D / J)^{2}\left(J / J_{0}\right)^{-2}>t^{\phi_{D / J}-\phi_{J / J}},
$$

i.e., if $\phi_{D / J}>\phi_{J / J_{0}}$. These exponents can be evaluat- 
ed from the equality (6). Taking $\beta_{\mathrm{SG}}=0.7, \beta_{\mathrm{RA}}=1.2$, and $\gamma_{0}=1.3$, we get $\phi_{J / J_{0}}=1.9$ and $\phi_{D / J}=2.4$. Random-anisotropy effects should dominate the critical properties of disordered systems if $(D / J)^{2}>t^{\phi_{D / J}}$ or $(D / J)^{2}>h^{\phi_{D / J} / \phi_{0}}$. Taking $\phi_{0}=1.4$, we get $D / J>t^{1.2}$ and $D / J>h^{0.85}$.

If critical exponents of random magnetic systems are interpreted in terms of a percolation mechanism of $\left\langle\left|S_{i} S_{j}\right|^{2}\right\rangle$ correlations, ${ }^{5,16}$ then hyperscaling leads to the fractal dimensionality $D \simeq 2.5$ for Heisenberg spin-glasses and $D \simeq 2$ for random-anisotropy systems. This suggests that different percolation mechanisms take place in these two types of systems.

We wish to thank J. P. Rebouillat and A. Lienard as well as P. Lethuillier and J. C. Genna for help in sample preparation and magnetization measurements.

${ }^{1}$ B. Dieny, thesis, University of Grenoble, 1985 (unpublished); B. Dieny and B. Barbara, J. Phys. (Paris) 46, 293 (1985), and Physica (Amsterdam) 130B, 245 (1985); J. Filippi, B. Dieny, and B. Barbara, Solid State Commun. 53, 6, 523 (1985).

2M. Suzuki, Prog. Theor. Phys. 58, 1151 (1977).

${ }^{3}$ K. H. Fischer, Phys. Rev. Lett. 34, 1438 (1975).

${ }^{4}$ A. Aharony and E. Pytte, Phys. Rev. B 27, 5872 (1983).

${ }^{5}$ B. Barbara and A. P. Malozemoff, J. Less-Common Metals 94, 45 (1983).

${ }^{6}$ A. P. Malozemoff, S. Barnes, and B. Barbara, Phys. Rev.
Lett. 51, 1704 (1983)

${ }^{7}$ D. S. Fisher and H. Sompolinsky, Phys. Rev. Lett. 54, 1063 (1985).

${ }^{8}$ B. Barbara, A. P. Malozemoff, and Y. Imry, Phys. Rev. Lett. 47, 1852 (1981).

${ }^{9}$ A. P. Malozemoff, Y. Imry, and B. Barbara, J. Appl. Phys. 53, 2205 (1982).

10P. Monod and H. Bouchiat, J. Phys. (Paris), Lett. 43, L45, (1982); H. Bouchiat, J. Phys. (Paris) 47, 71 (1986).

${ }^{11}$ The scattering of spin-glass exponents shown in Table I may result from inappropriate procedures in their determination, e.g., insufficient corrections for regular terms, or measurements performed out of the critical region or in ac fields. In the particular case of $a-\mathrm{GdAl}, \mathrm{dc}$ measurements have been used in the range of $t<0.15$ which corresponds to a reasonable width for the critical region of a spin-glass $[-\sqrt{ } Z$ times that of a ferromagnet, where $Z=10$ is the number of neighbors (Malozemoff, Imry, and Barbara, Ref. 9)]. Furthermore, it has been shown that critical exponents are almost insensitive to regular nonlinear contributions in this temperature range [B. Barbara, A. P. Malozemoff, and S. Barnes, J. Appl. Phys. 55, 1655 (1984)]

${ }^{12} \mathrm{G}$. Kotliar and H. Sompolinsky, Phys. Rev. Lett. 53, 1751 (1984).

13I. A. Campbell, N. de Courtenay, and A. Fert, J. Phys. (Paris), Lett. 45, L565 (1984), and Phys. Rev. B 30, 6791 (1984).

${ }^{14}$ Y. Yeshurun and H. Sompolinsky, Phys. Rev. Lett. 56, 984 (1986).

${ }^{15} \mathrm{~N}$. de Courtenay, H. Bouchiat, H. Hurdequint, and A. Fert, to be published.

${ }^{16}$ A. P. Malozemoff and B. Barbara, J. Appl. Phys. 57, 3410 (1985). 